\title{
Inferior sinus venosus defect: Echocardiographic diagnosis and surgical approach
}

\author{
Matthew A. Crystal, MD, ${ }^{a}$ Khaled Al Najashi, MD, ${ }^{\mathrm{b}}$ William G. Williams, MD, ${ }^{\mathrm{c}}$ Andrew N. Redington, MD, ${ }^{\mathrm{a}}$ and \\ Robert H. Anderson, $\mathrm{MD}^{\mathrm{d}}$
}

\begin{abstract}
Objective: We sought to define the inferior sinus venosus defect anatomically and document successful surgical approaches.
\end{abstract}

\begin{abstract}
Methods: We identified all patients previously given a diagnosis of an inferior sinus venosus defect at the Hospital for Sick Children, Toronto, Canada, between 1982 and 2005 by interrogating the cardiology and cardiac surgery databases. We included those having interatrial communications in which 1 or more of the right pulmonary veins drained to the inferior caval vein but retained connection with the left atrium, the rims of the oval fossa, and the walls of the coronary sinus, both being intact.
\end{abstract}
Results: We identified 11 children who had an interatrial communication meeting the criteria for and undergoing surgical repair of an inferior sinus venosus defect. Median age was 1.2 years; $6(55 \%)$ subjects were male, and none were cyanotic. Transthoracic echocardiographic analysis was performed preoperatively in all children, revealing right ventricular dilation in all. Surgical repair was accomplished with a pericardial patch. A complex baffle was needed in 3 children to maintain unobstructed inferior caval and pulmonary venous return. The echocardiographic diagnosis was complete in only 5 patients, but all diagnoses were correct since the year 2000. In all children the observations at surgical intervention showed that the defect was a venoatrial communi- cation involving drainage of the right pulmonary veins to the inferior caval vein while retaining connection to the left atrium.

Conclusions: Transthoracic echocardiographic analysis should remain the modality of choice for diagnosis of the inferior sinus venosus defect. We report excellent surgical results with a patch or baffle, correctly redirecting the anomalous venoatrial connections.

The inferior sinus venosus defect is a rare congenital cardiac malformation outside the confines of the normal atrial septum produced as a consequence of anomalous pulmonary venous connection to the inferior caval vein while retaining the pulmonary venous connection to the left atrium..$^{1-3}$ Therefore rather than representing an atrial septal defect, the lesion is better considered in terms of an anomalous venoatrial communication. Such venoatrial communications have been well delineated with respect to the superior caval vein. ${ }^{1,4}$ Those involving anomalous connection to the inferior caval vein, ${ }^{2}$ in contrast, are less well recognized. Thus far, diagnosis has been made either at the time of surgical repair or at autopsy. At present, there are no studies of large numbers of patients with such defects based on anatomic

\footnotetext{
From the Division of Cardiology, Department of Pediatrics, ${ }^{a}$ University of Toronto, the Hospital for Sick Children, Toronto, Ontario, Canada; the Division of Cardiolo-

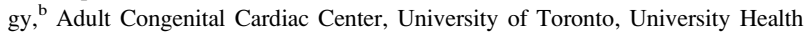
Network, Toronto, Ontario, Canada; the Division of Cardiovascular Surgery, Department of Surgery, ${ }^{\mathrm{c}}$ University of Toronto, the Hospital for Sick Children, Toronto, Ontario, Canada; and the Division of Cardiology, Department of Paediatrics, ${ }^{\mathrm{d}}$ Great Ormond Street Hospital, London, United Kingdom.

Received for publication May 15, 2008; revisions received Nov 5, 2008; accepted for publication Dec 19, 2008.

Address for reprints: Andrew N. Redington, MD, the Hospital for Sick Children, 555 University Ave, Toronto, Ontario, Canada M5G 1X8 (E-mail: andrew.redington@ sickkids.ca).

J Thorac Cardiovasc Surg 2009;137:1349-55

$0022-5223 / \$ 36.00$

Copyright (c) 2009 by The American Association for Thoracic Surgery

doi:10.1016/j.jtcvs.2008.12.010
}

diagnosis during life. Therefore we have sought to reinvestigate all patients identified with these defects during surgical correction at our institution. We have assessed the accuracy of the initial echocardiographic diagnosis, and we report the approaches used for surgical repair.

\section{MATERIALS AND METHODS}

\section{Study Population}

We identified all patients presumed to have an inferior sinus venosus defect at the Hospital for Sick Children, Toronto, Ontario, Canada, between July 1, 1982, and December 31, 2005. Our identification of such patients started with interrogation of the cardiology database to identify patients given diagnoses of an inferior sinus venosus defect. Of that group, there were very few who were coded correctly. Subsequently, given that all such patients undergo surgical intervention, we interrogated the surgical database. The initial approach was to cross-reference any child less than 18 years of age with an atrial septal defect, a partially anomalous pulmonary venous connection from the right lung, or both. This generated a list of 344 patients. From this list, we were able to identify 41 children in whom the surgical report described an interatrial communication in the environs of the inferior caval vein with or without partially anomalous pulmonary venous drainage. We examined the surgical reports of each of these patients to determine those patients with anatomy consistent with our understanding of the inferior sinus venosus defect, namely a venoatrial communication permitting shunting between the atrial chambers on the basis of anomalous pulmonary venous connection to the inferior caval vein with retention of the pulmonary venous connection to the left atrium. Using the surgical reports, we identified 11 children with this combination of findings. We then reviewed the medical records of these 11 patients. Ethical approval for the review was obtained from the Institutional Research Ethics Board of the Hospital for Sick Children. 


\begin{abstract}
Measurements
We collected demographic data, clinical symptoms at the time of presentation, electrocardiographic indices, and diagnostic imaging modalities used. Echocardiographic analysis had been performed in all children preoperatively by using the transthoracic approach in all, with none undergoing a preoperative transesophageal echocardiogram. Echocardiographic measurements, diagnoses, and general descriptors for each patient were recorded from the detailed final reports.
\end{abstract}

\section{Echocardiographic Data}

It is our institutional policy that all children undergo a complete transthoracic echocardiogram as their initial study so as to reduce the likelihood of incomplete or inaccurate diagnoses. All patients with interatrial communications undergo systematic recordings of the short- and long-axial subcostal and parasternal views, the apical views, and the suprasternal views by means of both cross-sectional and color Doppler interrogation, from which a description of the size and site of each defect is recorded. There was no attempt to rereview these studies for accuracy. Instead, we chose to rely on real-time diagnostic accuracy, as recorded in the final report, in the absence of an established surgical or anatomic diagnosis (see below). For all patients, it was the anatomic information gleaned from the surgical database, the surgical notes, or both that was used as the gold standard for diagnosis.

\section{Statistical Analysis}

Descriptive statistical analysis was used to describe the characteristics of the patients and the surgical results as medians with ranges and means with standard deviations.

\section{RESULTS \\ Anatomic Description}

The inferior sinus venosus defect produces shunting between the right and left atrial chambers in consequence of anomalous pulmonary venous connection to the inferior caval vein, whereby the anomalous pulmonary vein or veins retain their connection to the left atrium. This produces a venoatrial communication (Figure 1) that overrides the intact rims of the oval fossa (ie, fossa ovalis). The normal atrial septum is made up of the floor and rims of the oval fossa, with the superior and posterior rims being infoldings of the atrial walls between the attachments of the caval veins to the morphologically right atrium and the right pulmonary veins to the morphologically left atrium. With the inferior sinus venosus defect, the pulmonary veins have retained their connection to the left atrium while also having achieved continuity with the inferior caval vein. If the pulmonary veins did not maintain their connection to the left atrium, the inferior caval vein would connect only to the right atrium, resulting in standard partially anomalous pulmonary venous connection to the inferior caval vein or inferior right atrium. It is because the pulmonary veins have attained the anomalous connection to the right atrium or its systemic tributaries yet retained their continuity with the posteroinferior wall of the left atrium that there is an extraseptal venoatrial confluence that permits the interatrial shunting. Therefore the essence of the inferior sinus venosus defect is a venoatrial connection of the pulmonary and inferior caval veins that is in continuity with both the right and left atrial walls.
The confluence thus created is outside the boundaries of the normal atrial septum; in other words, it is an extraseptal interatrial communication. As shown in Figure 1, the venoatrial communication is outside the confines of the oval fossa, which can itself be intact, be probe patent, or have a deficient floor permitting additional interatrial shunting. In the heart shown in Figure 1, A, which is held in the museum of Pittsburgh Children's Hospital, there is a persistent left superior caval vein draining to the right atrium through an enlarged coronary sinus. In the heart shown in Figure $1, B$, from our own museum, there is also a persistent left superior caval vein opening to the coronary sinus, along with anomalous connection of the right superior pulmonary vein directly to the right atrium in the absence of a right superior caval vein. In both hearts the anomalous inferior venoatrial communication overrides the intact posteroinferior rim of the oval fossa. The association with persistent patency of the left superior caval vein is likely to be a chance finding. It should not be anticipated always to exist in patients with inferior sinus venosus defects.

\section{Clinical Information}

At the time of the operation, all the children included in our study were found to have comparable anatomic findings to the arrangement shown in Figure 1, with the exception of persistent patency of the left superior caval vein, which was not found in any of our patients. Of these patients, $6(55 \%)$ were boys. At the diagnosis, median age was 1.2 years, with a range from 0.25 to 3.5 years. The initial median height was $81.9 \mathrm{~cm}$, with a range of 61 to $102 \mathrm{~cm}$, and the median weight was $9.9 \mathrm{~kg}$, with a range of 5.9 to $16.0 \mathrm{~kg}$. Blood pressure and heart rate were normal in all children. None of the children demonstrated cyanosis or desaturation as identified with the saturations of oxygen by means of pulse oximetry, this being recorded as between $97 \%$ and $100 \%$ in the most recent 6 patients for whom this test was available. All children had normal jugular venous pulsations, and none presented with respiratory distress. Hepatosplenomegaly was identified in only 1 child. As expected, all children demonstrated a widely split second heart sound and a systolic ejection murmur. Additionally, a middiastolic rumble was audible in 5 patients, with a right ventricular heave also palpable in 5 patients.

\section{Diagnostic Tests}

A scalar electrocardiogram was obtained preoperatively in 10 patients, all of whom had normal sinus rhythm. All children were found to have normal conduction, depolarization, and repolarization. By using voltage criteria, right atrial enlargement was diagnosed in 7 children, left atrial enlargement in 2 children, and right ventricular hypertrophy in 2 children, with none showing evidence of left ventricular hypertrophy. There were no instances of arrhythmia or any evidence of ischemic changes. 

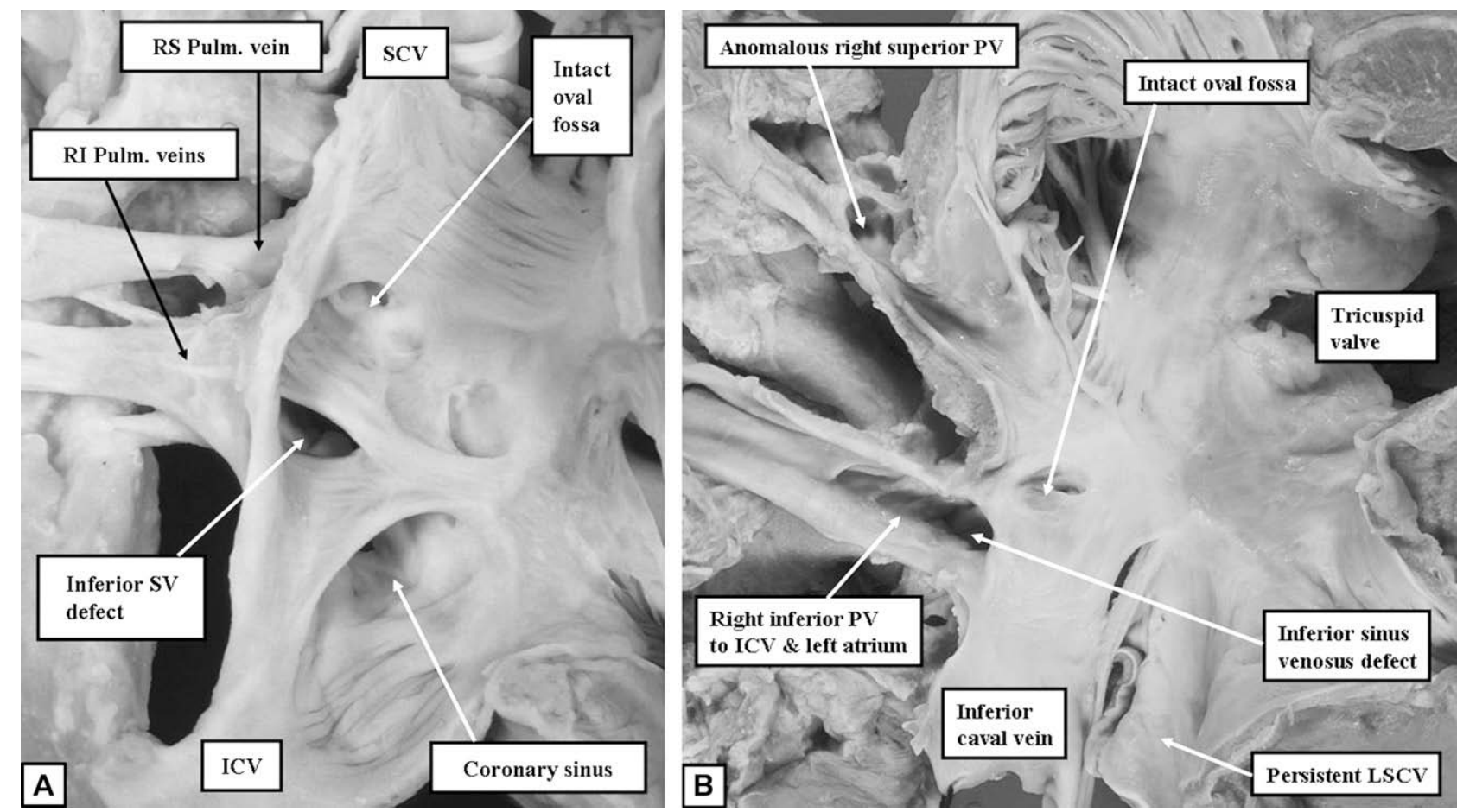

FIGURE 1. Photographs of a pathologic specimen from the archive of Pittsburgh Children's Hospital (A) and a specimen from the collection at the Hospital for Sick Children (B) demonstrating an inferior sinus venosus defect, a venoatrial communication outside the confines of an intact oval fossa. In A, the arrows point to the outside of the pulmonary veins. In B, the arrows for the inferior pulmonary vein and the defect point to the same hole. This hole exists because the inferior pulmonary vein is connected to the inferior caval vein while retaining its connection to the left atrium. RS pulm. vein, Right superior pulmonary vein; $S C V$, superior caval vein; RI pulm. veins, right inferior pulmonary veins; $S V$, sinus venosus; $I C V$, inferior caval vein; $P V$, pulmonary vein; $L S C V$, left superior caval vein.

\section{Echocardiographic Data}

Ventricular function was normal for all children, with a mean left ventricular ejection fraction of $73 \% \pm 12 \%$, and a mean shortening fraction of $41 \% \pm 11 \%$. Right ventricular dilation was identified in all children, with a mean right ventricular end-diastolic dimension of $2.2 \pm 0.7 \mathrm{~cm}$ (mean $z$ score, $5.1 \pm 1.4$ ). In Table 1 we summarize the diagnoses identified on the preoperative echocardiograms, and in Table 2 we summarize the presumed connection of the caval and pulmonary veins. Figure 2 demonstrates the typical echocardiographic images of an inferior sinus venosus defect from the subcostal view. None of our patients underwent an echocardiographic bubble contrast study, cardiac catheterization, or cardiac magnetic resonance imaging.

\section{Surgical Repair}

The median age at the time of surgical intervention was 2.8 years, with a range of 1.3 to 6.8 years, and median weight was $14.8 \mathrm{~kg}$, with a range of 9.5 to $20.8 \mathrm{~kg}$. The surgical repair was accomplished with a pericardial patch of autologous material in 8 patients and a xenograft in 3 patients, which was placed to ensure unobstructed postoperative connection of the pulmonary veins to the left atrium and the inferior caval vein to the right atrium. So as to achieve an unobstructed connection of the inferior caval vein, it was necessary in 3 children to construct a baffle of complex shape by molding a simple patch to ensure the pulmonary vein and caval vein drained appropriately without stenosis. Reimplantation of the individual pulmonary veins was not required. For those children in whom it was deemed that a baffle was needed to reconnect the pulmonary veins to the left atrium, the preliminary step was to create an interatrial communication by excising the posteroinferior rim of the oval fossa, thus extending the initial venoatrial communication relative to the mouth of the inferior caval vein. If patent, the oval fossa itself was closed with direct sutures.

An appropriate preoperative echocardiographic diagnosis with recognition of the venoatrial communication had been made in only 5 of 11 patients, although the presence of an inferior sinus venosus defect had been suspected in 1 additional patient (Table 3); all have received correct diagnoses, however, since the year 2000. The anomalous pulmonary venous connections to the inferior caval vein had not been completely identified by means of preoperative echocardiographic analysis (Table 4). All patients underwent complete surgical repair without major complications, and there was no instance of postoperative cyanosis or caval or pulmonary 
TABLE 1. Preoperative echocardiographic diagnoses

\begin{tabular}{|c|c|c|c|c|}
\hline Patient no. & Age at diagnosis (y) & Echocardiographic diagnosis & Pulmonary venous connection & Associated defects \\
\hline 1 & 3.5 & $2^{\circ} \mathrm{ASD}, \mathrm{RAE}, \mathrm{RV}$ dilation & & \\
\hline 2 & 1.08 & Large $2^{\circ}$ ASD; paradoxic septal motion & PAPVC RSPV (?RMPV to RA) & \\
\hline 3 & 0.42 & $2^{\circ} \mathrm{ASD}$ & & Valvar PS \\
\hline 4 & 0.25 & SVD-inferior, RV dilation & PAPVC-R pulmonary veins to RA & \\
\hline 5 & 1 & $2^{\circ} \mathrm{ASD}$ & & \\
\hline 6 & 1.5 & $2^{\circ} \mathrm{ASD}$ & & \\
\hline 7 & 0.5 & Large posteroinferior $2^{\circ} \mathrm{ASD}$ vs SVD-inferior & PAPVC & Anomalous LSCA, RAA \\
\hline 8 & 0.25 & SVD-inferior & PAPVC & \\
\hline 9 & 0.17 & SVD-inferior & & \\
\hline 10 & 3.5 & SVD-inferior & & LSVC-CS \\
\hline 11 & 0.92 & SVD-inferior & R PAPVC-RSPV to SCV-RA & PMVSD, PS, PFO \\
\hline
\end{tabular}

venous obstruction, as determined by means of echocardiographic analysis and overall clinical well-being. All children maintained good ventricular function without any long-term caval or pulmonary venous obstruction. In the immediate postoperative period, 2 children demonstrated acceleration of flow within the right pulmonary veins, but all demonstrated normal flow on subsequent studies. In 3 of the children, small pericardial effusions had been identified postoperatively by means of echocardiographic analysis, with none having any hemodynamic instability. Not all the children have been followed postoperatively at our institution for extended periods of time. Those referred for surgical repair by external cardiologists were only followed until the time of discharge. Those referred from within the hospital, in contrast, were followed until they reached the age of 18 years, with the duration of this follow-up ranging from 3 days to 12.9 years. Follow-up data for all patients were included until the most recent echocardiogram. That being said, all patients remain clinically well without signs of obstruction, and none have required reoperation or reintervention.

TABLE 2. Connections of the caval veins and pulmonary veins as diagnosed with preoperative echocardiographic analysis

\begin{tabular}{lrrc}
\hline Venous structure & RA & LA & Both \\
\hline RSPV & 5 & 6 & 0 \\
RMPV & 1 & 3 & 0 \\
RIPV & 4 & 6 & 1 \\
LSPV & 0 & 11 & 0 \\
LIPV & 0 & 11 & 0 \\
RSCV & 11 & 0 & 0 \\
ICV & 11 & 0 & 0 \\
\hline
\end{tabular}

$R A$, Right atrium; $L A$, left atrium; $R S P V$, right superior pulmonary vein; $R M P V$, right middle pulmonary vein; $R I P V$, right inferior pulmonary vein; $L S P V$, left superior pulmonary vein; $L I P V$, left inferior pulmonary vein; $R S C V$, right superior caval vein; $I C V$, inferior caval vein.

\section{DISCUSSION}

To the best of our knowledge, ours is the largest group of patients thus far reported with an inferior sinus venosus defect. Clear anatomic criteria are needed to differentiate this defect from others within the oval fossa. To this end, as suggested by others, ${ }^{2,5}$ we initially considered the inferior sinus venosus defect to represent an interatrial communication bordered directly by the inferior caval and right inferior

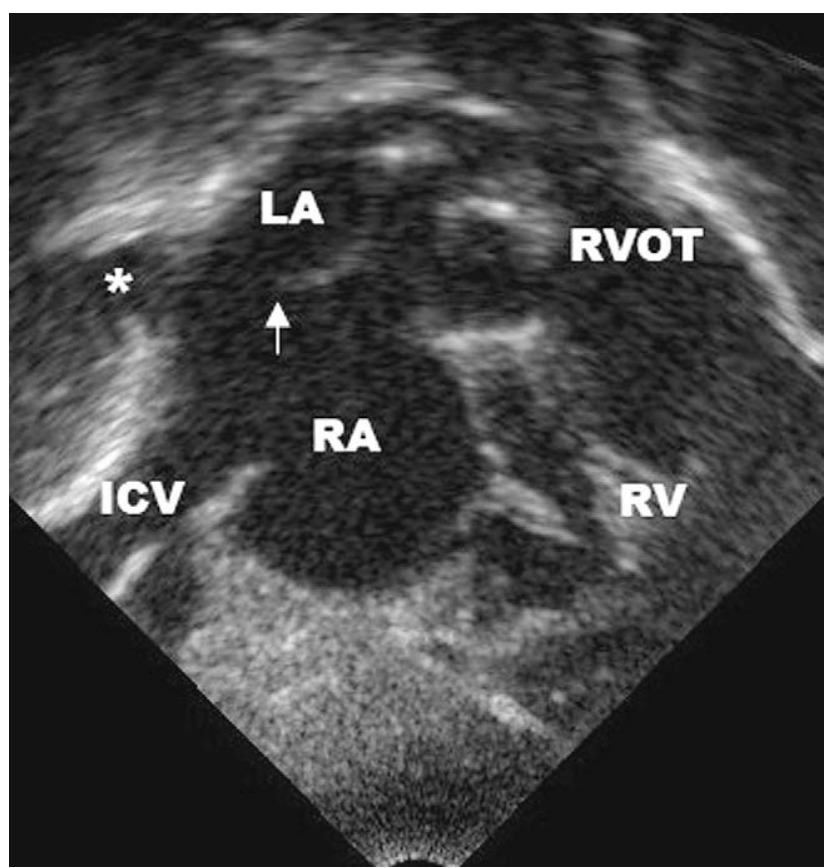

FIGURE 2. The image demonstrates the echocardiographic subcostal right anterior oblique view of the inferior sinus venosus defect. The white arrow points at the most inferior margin of the intact atrial septum and marks the roof of the venoatrial communication. The asterisk is placed within the right inferior pulmonary vein and demonstrates the anomalous connection to the right atrium. $L A$, Left atrium; $R V O T$, right ventricular outflow tract; $I C V$, inferior caval vein; $R A$, right atrium; $R V$, right ventricle. 
TABLE 3. Postoperative cardiac diagnosis (confirmed at operation)

\begin{tabular}{|c|c|c|c|c|}
\hline $\begin{array}{l}\text { Patient } \\
\text { no. }\end{array}$ & $\begin{array}{l}\text { Age at } \\
\text { eeration } \\
(y)\end{array}$ & Diagnosis & $\begin{array}{l}\text { Pulmonary } \\
\text { venous } \\
\text { connection }\end{array}$ & $\begin{array}{l}\text { Associated } \\
\text { defects }\end{array}$ \\
\hline 1 & 6.8 & SVD-inferior & PAPVC & $2^{\circ} \mathrm{ASD}$ \\
\hline 2 & 4.8 & SVD-inferior & PAPVC-RSPV/RIPV & \\
\hline 3 & 3.9 & SVD-inferior & & PFO, no PS \\
\hline 4 & 2.7 & SVD-inferior & $\begin{array}{l}\text { PAPVC-RIPV/ } \\
\text { RMPV }\end{array}$ & \\
\hline 5 & 1.5 & SVD-inferior & & \\
\hline 6 & 1.8 & SVD-inferior & & \\
\hline 7 & 2.8 & SVD-inferior & $\begin{array}{l}\text { PAPVC-right } \\
\text { pulmonary veins }\end{array}$ & $\begin{array}{l}\text { RAA with } \\
\text { vascular ring }\end{array}$ \\
\hline 8 & 1.3 & SVD-inferior & PAPVC-RIPV & \\
\hline 9 & 4.2 & SVD-inferior & & PFO \\
\hline 10 & 4.9 & SVD-inferior & PAPVC-RIPV & PFO \\
\hline 11 & 1.5 & SVD-inferior & $\begin{array}{l}\text { PAPVC-right } \\
\text { pulmonary veins }\end{array}$ & $\begin{array}{l}\text { Valvar PS, } \\
\text { PMVSD }\end{array}$ \\
\hline
\end{tabular}

Note that, despite the location of the pulmonary veins, in all patients the surgeon discovered an extraseptal cavopulmonary confluence that permitted left-to-right interatrial shunting. $S V D$, Sinus venosus defect; $2^{\circ} A S D$, interatrial communication at secondary septum; $P A P V C$, partial anomalous pulmonary venous connection; $R S P V$, right superior pulmonary vein; $R I P V$, right inferior pulmonary vein; $P F O$, patent oval foramen; $P S$, pulmonary stenosis; $R M P V$, right middle pulmonary vein; $R A A$, right aortic arch; $R A E$, right atrial enlargement; $P M V S D$, perimembranous ventricular septal defect.

pulmonary veins in the setting of intact rims of the oval fossa and a patent coronary sinus with no fenestrations of its walls. At recent discussions of the International Nomenclature Committee, however, it was pointed out that rather than representing an interatrial communication, the defect was an anomalous venoatrial communication, the essential feature being anomalous pulmonary venous connection to the inferior caval vein with retention of pulmonary venous connection to the left atrium. This combination produces an extraseptal cavopulmonary conduit, permitting shunting of blood from the left to the right atrium. Such a definition excludes the inferior sinus venosus defect from interatrial communications existing through the mouth of the coronary sinus or true septal defects within the oval fossa extending posteroinferiorly into the mouth of the inferior caval vein. Even with the use of this anatomic criterion, appropriate diagnosis of the defect remains difficult during life, as noted in studies comparing echocardiographic findings with anatomic specimens ${ }^{6}$ and as shown by our own experience, wherein the preoperative diagnosis was incomplete in 6 of 11 children. We anticipate that the diagnosis would have been made with greater frequency had those investigating the patients been aware that the lesion was a venoatrial malformation rather than a purely interatrial communication, as demonstrated by our recent echocardiographic results.

The crucial anatomic factor in diagnosis, either echocardiographically or morphologically, is the establishment of the integrity of the rims of the oval fossa. ${ }^{2,4}$ The description of the defect as given by the surgeon is important in making this distinction, as it is in defining the specific location of the
TABLE 4. Connections of the caval veins and pulmonary veins (confirmed at operation)

\begin{tabular}{lrrc}
\hline Venous structure & RA & LA & Both \\
\hline RSPV & 6 & 4 & 0 \\
RMPV & 5 & 3 & 0 \\
RIPV & 3 & 8 & 0 \\
LSPV & 0 & 11 & 0 \\
LIPV & 0 & 11 & 0 \\
RSCV & 11 & 0 & 0 \\
ICV & 6 & 3 & 1 \\
\hline
\end{tabular}

As described in the legend to Table 3, it should be noted that in all patients the surgeon found an extraseptal cavopulmonary confluence that permitted left-to-right interatrial shunting. $R A$, Right atrium; $L A$, left atrium; $R S P V$, right superior pulmonary vein; $R M P V$, right middle pulmonary vein; $R I P V$, right inferior pulmonary vein; $L S P V$, left superior pulmonary vein; $L I P V$, left inferior pulmonary vein; $R S C V$, right superior caval vein; $I C V$, inferior caval vein.

communication and the precise connections of the inferior caval vein and the right pulmonary veins. Recognition of the overriding mouth of the anomalous systemic-pulmonary venous confluence allows the surgeon either to patch or to baffle the pulmonary and caval pathways to the appropriate atrium without causing obstruction or permitting a residual shunt. In our series all patients had the venous connections incorporated into the appropriate atriums. This was achieved with a simple patch in 8 instances and a more complex baffle in 3 instances. In no patient was an impediment created relative to the venous streams, nor was obstruction or cyanosis present postoperatively.

\section{Diagnostic Accuracy}

The essential feature of the inferior sinus venosus defect is the anomalous pulmonary venous connection to the inferior caval vein with retention of the normal pulmonary venous connection to the left atrium. In all of our patients, the left pulmonary veins were normally connected to the left atrium, but as expected, some of the right pulmonary veins were anomalously connected. Although some of the pulmonary veins are connected abnormally, this does not necessarily mean that they drain anomalously. This is because the inferior caval vein itself attains a left atrial connection by virtue of the creation of the anomalous venoatrial communication. Indeed, in only 3 of our patients were the pulmonary veins positioned so as to drain directly into the right atrium. The inferior caval vein, rather than draining directly to the right atrium, was deemed at operation in 4 of our patients to be draining either directly to the left atrium or to both atriums. Nonetheless, the clinical picture remains that of a left-toright shunt, and a right-to-left shunt was not seen in any patient. The hemodynamic abnormality is the same as all instances in which there is shunting between the atriums, and therefore it is not possible based on clinical features to differentiate the inferior sinus venosus defect from other forms of interatrial communications. Therefore it is the 
responsibility of the cardiologist to define the anatomy in preparation for a surgical repair. At our institution, all patients undergo transthoracic echocardiographic analysis as the standard baseline investigation.

In our patients the appropriate nature of the inferior sinus venosus defect was correctly diagnosed in only half our cohort by means of echocardiographic analysis, with accuracy increasing since the year 2000, after which all patients have been correctly identified. We presume that this improved diagnostic accuracy reflects improved technology along with our better understanding of the nature of these defects. The anomalous venoatrial connections identified by means of echocardiographic analysis were verified at the time of the operation, but an additional 7 anomalous connections were found that had not been diagnosed preoperatively. The incomplete diagnosis did not affect surgical outcome, and we anticipate that these findings will be diagnosed in the future in light of our increased understanding of the anatomic complexity. The associated anomalies were all confirmed at the time of the operation, except for the diagnosis of valvar pulmonary stenosis, with a normal valve being observed at the time of the operation. In young children and in others with good acoustic windows, we anticipate that transthoracic echocardiographic analysis will provide, in the majority of cases, the pertinent information for appropriate operative planning, as already suggested by others. ${ }^{7}$

In this era of catheter-based closure of atrial septal defects, correct identification of this type of defect avoids the obligate pulmonary venous obstruction that would be created by using a device for occlusion.

There is a trend toward more invasive and expensive testing for patients with this diagnosis because accuracy of all features remains a challenge. Transesophageal echocardiographic analysis offers excellent acoustic windows and is ideally suited to defining the posterior features and connections but is limited in its accessibility to patients, especially children in the preoperative awake setting. ${ }^{8,9}$ Intraoperative transesophageal echocardiographic analysis at completion of surgical repair allows for immediate and early detection of residual defects, obstructed caval or pulmonary venous pathways, and confirmation of cardiac anatomy. We routinely use intraoperative transesophageal echocardiographic analysis at our institution. Kronzon and colleagues ${ }^{10}$ demonstrated that diagnosis of interatrial communications using transesophageal echocardiographic analysis is superior to the transthoracic approach in adults, with only one quarter of the defects being correctly diagnosed. The majority of diagnostic difficulties arose from the poor acoustic windows and therefore might not be as relevant in children. Magnetic resonance imaging offers excellent anatomic imaging and diagnostic accuracy, as well as a greater ability to define associated lesions. ${ }^{11,12}$ Because of the requirement of general anesthesia for high-quality acquisition of images in young children, it is our opinion that magnetic resonance imaging should have a limited role, being used only when surgical intervention is not certain. Cardiac catheterization and computed tomographic analysis offer increased diagnostic accuracy when compared with clinical findings and transthoracic echocardiographic analysis, but the additional radiation and invasiveness is neither required nor recommended based on other available modalities. ${ }^{13,14}$

\section{Surgical Approach}

A number of authors have outlined different approaches to surgical correction of this rare anomaly. Reimplantation of the anomalously connected pulmonary vein or veins directly into the left atrium, combined with suture closure of the venoatrial communication, has been successfully reported. ${ }^{15}$ Others have reported the detachment of the posterior edge of the atrial septum, suturing it into position to the right of the anomalously connected pulmonary vein and leftward of the caval veins, thereby closing the left-to-right defect and thus redirecting the anomalous pulmonary venous return to the left atrium without requiring a patch. ${ }^{16,17}$ In our institution a strategy toward simple repair with a patch or baffle avoids the potential long-term venous obstruction associated with the aforementioned approaches. We used a baffle rather than a simple patch when we considered the insertion of the patch might induce either caval or pulmonary venous obstruction.

\section{CONCLUSION}

The inferior sinus venosus defect is best defined as a venoatrial communication existing in the setting of intact rims of the atrial septum itself produced in consequence of anomalous pulmonary venous connection to the inferior caval vein with retention of the pulmonary venous connection to the left atrium. In children, despite its previously poor yield, we still believe that transthoracic echocardiographic analysis remains the imaging modality of choice and that responsibility for recognizing the inferior sinus venosus defect is vested in the cardiologist. Differentiation of an inferior sinus venosus defect from a defect within the oval fossa is necessary to refer appropriately and directly for surgical intervention. Recognition of the anomalous pulmonary connections then becomes the responsibility of the surgeon, both for diagnosis and appropriate repair. There are multiple surgical approaches that have been used. We report excellent results with a pericardial patch or baffle as our routine strategy for direct repair.

\section{References}

1. Al Zaghal AM, Li J, Anderson RH, Lincoln C, Shore D, Rigby ML. Anatomical criteria for the diagnosis of sinus venosus defects. Heart. 1997;78:298-304.

2. Ettedgui JA, Siewers RD, Zuberbuhler JR, Anderson RH. Echocardiographic diagnosis of inferior sinus venosus defects. Cardiol Young. 1992;2:338-41.

3. Wong DR, Hancock Friesen CL, Warren AE, O'Blenes SB. Echocardiographic diagnosis and repair of an inferior sinus venosus defect. Can J Cardiol. 2008; $24: 67$. 
4. Li J, Al Zaghal AM, Anderson RH. The nature of the superior sinus venosus defect. Clin Anat. 1998;11:349-52.

5. Munoz-Castellanos L, Espinola-Zavaleta N, Kuri-Niyon M, Ruiz JF, Keirns C. Atrial septal defect: anatamoechocardiographic correlation. J Am Soc Echocardiogr. 2006;19:1182-9.

6. Van Praagh S, Carrera ME, Sanders SP, Mayers JE, Van Praagh R. Sinus venosus defects: unroofing of the right pulmonary veins-anatomic and echocardiographic findings and surgical treatment. Am Heart J. 1994;128:365-79.

7. Muhler EG, Engelhardt W, von Bernuth G. Detection of sinus venosus atrial septal defect by two-dimensional echocardiography. Eur Heart J. 1992;13:453-6.

8. Gnanapragasam JP, Houston AB, Northridge DB, Jamieson MP, Pollock JC. Transoesophageal echocardiographic assessment of primum, secundum and sinus venosus atrial septal defects. Int J Cardiol. 1991;31:167-74.

9. Oliver JM, Gallego P, Gonzalez A, Dominguez FJ, Aroca A, Mesa JM. Sinus venosus syndrome: atrial septal defect or anomalous venous connection? A multiplane transoesophageal approach. Heart. 2002;88:634-8.

10. Kronzon I, Tunick PA, Freedberg RS, Trehan N, Rosenzweig BP, Schwinger ME. Transesophageal echocardiography is superior to transthoracic echocardiography in the diagnosis of sinus venosus atrial septal defect. J Am Coll Cardiol. 1991;17:537-42.
11. Valente AM, Sena L, Powell AJ, Del Nido PJ, Geva T. Cardiac magnetic resonance imaging evaluation of sinus venosus defects: comparison to surgical findings. Pediatr Cardiol. 2007;28:51-6.

12. Kessel-Schaefer A, Linka A, Pretre R, Buser P. Inferior sinus venosus defect associated with incomplete cor triatriatum dexter and patent foramen ovale. Eur Soc Cardiol. 2006;7:239-42.

13. Freed MD, Nadas AS, Norwood WI, Castaneda AR. Is routine preoperative cardiac catheterization necessary before repair of secundum and sinus venosus atrial septal defects. J Am Coll Cardiol. 1984;4:333-6.

14. Otsuka M, Itoh A, Haze K. Sinus venosus type of atrial septal defect with partial anomalous pulmonary venous return evaluated by multislice CT. Heart. 2004;90: 901.

15. Perna AM, Alajmo F. Partial anomalous pulmonary venous return of an uncommon type. J Cardiovasc Surg. 1984;25:563-5.

16. Sturm JT, Ankeney JL. Surgical repair of inferior sinus venosus atrial septal defect. J Thorac Cardiovasc Surg. 1979;78:570-2.

17. Hiramatsu T, Takanashi Y Imai Y, Hoshino S, Seo K, Terada M, et al. Atrial septa displacement for repair of anomalous pulmonary venous return into the right atrium. Ann Thorac Surg. 1998;65:1110-4 